www.jmscr.igmpublication.org

Index Copernicus Value: 79.54

ISSN (e)-2347-176x ISSN (p) 2455-0450

crossrefDOI: https://dx.doi.org/10.18535/jmscr/v7i1.196

\title{
Study of Relationship between Different Phases of Menstual Cycle and Audiovisual Reaction Time in Healthy Females
} Corresponding Author

Anju Kumari

Lady Medical Officer, Indira Gandhi Institute of Medical Sciences, Sheikhpura, Patna, India Email:anju81114@gmail.com, Phone: 9693866311

\begin{abstract}
Objectives: Menstrual cycle is due to cyclic variations in the levels of various sex hormones.

This study was done to evaluate the influence of menstrual cycle on auditory and visual reaction times.

Material and Methods: This study was conducted on 50 healthy young females in the age group of 18-25 years, having regular menstrual cycles. The Auditory and Visual reaction time were measured by indigenously prepared reaction time software in menstrual, proliferative, and secretory phases.

Results: The average reaction times (in milliseconds) during the different phases of the menstrual cycle were as follows: $A R T=M-198.28 \pm 14.688, P-192.58 \pm 16.63, S-185.47 \pm 13.27$ and $V R T=M-$ $246.13 \pm 8.21, P-238.36 \pm 10.32, S-232.87 \pm 6.38$.

Conclusion: Both the ART and VRT were longer during the menstrual phase, whereas they were shorter during the secretory phase of the menstrual cycle which could be due to changes in the levels of female sex hormones.

Keywords: reaction time, auditory, visual, sex hormones.
\end{abstract}

\section{Introduction}

The biological activity of the menstrual cycle is created by the coordination among hypothalamic, hypophyseal and ovarian hormones ${ }^{1}$. Menstrual cycle refers to the cyclic changes which occur in the endometrium during active reproductive years in females, leading to a recurrent monthly bleeding per vaginum. Menstrual cycle of 28 dayscan be divided into 3 phases, menstrual, proliferative (follicular), and luteal (secretory). About 24 hours before the end of menstrual cycle, oestrogen and progesterone levels decline, resulting in menstrual bleeding. This is followed by a proliferative phase which is under the influence of oestrogen and then the secretory phase, which is under the influence of oestrogen and progesterone ${ }^{2}$. 
The whole menstrual cycle is governed by a wellcoordinated and appropriately regulated changes in the levels of ovarian steroid hormones (estrogen and progesterone), which are responsible for producing the discrete responses in multiple tissues andorgans ${ }^{3}$.

Sex hormones are known to affect various CNS modalities such as neurite outgrowth, synaptogenesis, dendritic branching, myelination which ultimately affects the neural plasticity ${ }^{4}$.

The reaction time is the time interval between the stimulus application and the appropriate voluntary response as rapidly as possible ${ }^{5}$. It involves stimulus processing, decision making and response programming.

\section{Materials and Methods}

The study was conducted in the Department of Physiology, Vardhman Institute of Medical Sciences, Pawapuri, Bihar with prior approval from Ethical committee. The subjects for this study were selected from normal healthy females in the age group of 18-25 years, having regular menstrual cycles. 50 subjects who satisfied the inclusion and exclusion criteria were selected,and informed consent was obtained from them.

The purpose, procedure and noninvasive nature of the study were explained and written informed consent for the study was taken from each subject. Height (meters) and weight $(\mathrm{kg})$ of each subject were noted.

The subjects, who were included in the study, were healthy unmarried females in the specified age group of 18-25 years, having regular menstrual cycles of 27-30 days for at least the last 6 months, and whose body mass index was within the normal range $\left(18.5-24.9 \mathrm{~kg} / \mathrm{m}^{2}\right)$. The subjects, taking any medication or hormonal preparations, those having any physical illness orendocrinological disorders, smokers, alcoholics, athletes orthose involved in excessive physical activity were excluded from the study.

The subjects were instructed to visit the department during each phase of the menstrual cycle. $1-5^{\text {th }}$ day, $9-12^{\text {th }}$ day, and $19-22^{\text {nd }}$ day were selected to represent menstrual, proliferative, and secretory phases, respectively according to daily basal body temperature recordings.

Visual Reaction Time and Auditory Reaction Time were measured by indigenously prepared Reaction Time Software. The visual stimulus consists of Colored Square that flashes in the center of the screen. The auditory stimulus consists of a computer generated beep of $3000 \mathrm{~Hz}$ / $200 \mathrm{msec}$, presented via a headphone to both ears. The frequency and duration of the stimulus were kept constant over sequential trials but the duration between two beeps varied randomly in order to prevent bias prior anticipation.

All the subjects were subjected to ART and VRT recording in a quiet room in the Department of Physiology, Vardhman Institute of Medical Sciences, Pawapuri, Bihar. The subjects were asked to sit in front of the computer screen and to put the index finger of their dominant hand lightly on the "Enter" button of the keyboard of the computer. They were asked to press the "Enter" button as quickly as possible when a visual or auditory stimulus was presented to them. Three consecutive readings of each stimulus were recorded. The lowest reading was taken as reaction time.

\section{Result}

The age, height, weight and BMI of subjects are shown in Table 1. The average values (in milliseconds) of the ART and VRT in different phases of the menstrual cycle are shown in Table 2. Comparison between various phases of the menstrual cycle for ART and VRT are shown in Table 3.

Table No. 1: Age, Anthropometric data and Body Mass Index (BMI) of subjects

\begin{tabular}{|l|c|}
\hline PARAMETERS & \\
\hline Age $(\mathrm{yrs})$ & $21.53 \pm 0.693$ \\
\hline Height $(\mathrm{cm})$ & $153.3 \pm 3.27$ \\
\hline Weight $(\mathrm{kg})$ & $54.06 \pm 4.03$ \\
\hline BMI $\left(\mathrm{kg} / \mathrm{m}^{2}\right)$ & $22.397 \pm 1.1512$ \\
\hline
\end{tabular}


Table No. 2: Auditory and Visual Reaction Time in different phases of menstrual cycle

\begin{tabular}{|l|l|l|}
\hline Phase & ART $(\mathrm{msec})$ & VRT $(\mathrm{msec})$ \\
\hline Menstual & $198.28 \pm 14.688$ & $246.13 \pm 8.21$ \\
\hline Proliferative & $192.58 \pm 16.63$ & $238.36 \pm 10.32$ \\
\hline Secretory & $185.47 \pm 13.27$ & $232.87 \pm 6.38$ \\
\hline
\end{tabular}

Table No. 3: Comparison between various phases of the menstrual cycle for ART and VRT

\begin{tabular}{|l|c|c|c|c|}
\hline $\begin{array}{l}\text { Phase } \\
\text { pairs }\end{array}$ & ART & & VRT & \\
\hline & t value & p value & t value & p value \\
\hline I and II & 4.6653 & $<0.001^{*}$ & 4.7821 & $<0.001^{*}$ \\
\hline II and III & 4.7261 & $<0.001^{*}$ & 4.5860 & $<0.001^{*}$ \\
\hline I and III & 5.0613 & $<0.001^{*}$ & 5.0367 & $<0.001^{*}$ \\
\hline
\end{tabular}

Phase-I: Menstrual phase, Phase-II: Proliferative phase, Phase-III: Secretory phase, *Very highly significant, ART: Auditoryreaction time, VRT: Visual reaction time

Statistical analysis: All results were expressed in Mean \pm S.D. Mean and Standard Deviation of all parameters were calculated according to accepted statistical methods. The statistical tests used were ANOVA and Students ' $t$ ' test. Data was analysed with the aid of appropriate software.

\section{Discussion}

The sex hormones, especially estrogen and progesterone secreted from the ovaries, vary in their level during different phases of menstrual cycle. The menstrual phase is characterized by low levels of both these hormones and as we progress through the follicular phase, estrogen level rises rapidly to reach the peak just before ovulation, with progesterone levels continuing to remain low. Whereas during the luteal phase, the levels of both estrogen and progesterone rises, thus in the mid luteal phase, both the hormones are high. Hence during the normal menstrual cycle, there are two peaks of estrogen secretion, an "ovulation peak," which occurs near the end ofthe follicular phase and a "luteal peak." Progesterone reachesa peak about 4-7 days before menstruation ${ }^{6}$.

A study by Smith et al. showed for the first time that the activities of a specific neural network composed of excitatory afferents and inhibitory interneurons, that ultimately determines the excitability of corticospinal neurons, is not constant through the various phases of a normal menstrual cycle ${ }^{7}$.

While some studies have observed lower threshold for auditory stimuli during the beginning of the menstrualperiod ${ }^{8}$, in our study, the ART and VRT were longer in the menstrual phase and shorter in secretory phase as compared to the proliferative phase.

In a study conducted by Nene and Pazare, ART was significantly increased in premenstrual phase and on the expected day of ovulation ${ }^{9}$. Similarly in a study conducted by Das et al., there was a significant increase in weight as well as prolongation in auditory and visual Response times during the premenstrual phase. These changes have been attributed to fluid and salt retention occurring in this phase, which inturn leads to a decrease in the processing capability of central nervous system ${ }^{10}$. Bhatia et al. observed a higher threshold for evoking the $\mathrm{V}$ wave of BAEP and a higher absolute peak latency of waves with a decrease in amplitude of wave Vduring premenstrual phase and have attributed this to the delayed conduction time of auditory impulses from auditory nerve to midbrain ${ }^{11}$.

Bruce and Russel, have suggested that retention of water and sodium due to variations in the levels of sex steroids during the menstrual cycle, might influence either the process of axonal conduction time or the availability of neurotransmitters at synapses in the auditory pathways ${ }^{12}$.

A study by Afshan et al. has found that the shortest VRT occur during the luteal phase ${ }^{13}$. The most likely cause for this is that estrogen causes delay in conduction by influencing gamma amino butyric acid release at the various polysensory association areas of brain, which is blunted by the presence of progesterone ${ }^{14}$.

\section{Conclusion}

Shorter reaction time was observed during the secretory phase, which is probably due to the higher levels of progesterone seen in this phase. The menstrual phase is characterized by longer 
reaction times, which could be due to a generalized slowing of neural conduction times, because of fluid and salt retention.

\section{Conflict of Interest: None \\ Source of Funding: Self}

\section{References}

1. Cooke WH, Ludwig DA, Hogg PS, Eckburg DL, Convertino VA. Does the menstrual cycle influence the sensitivity ofvagally mediated baroreflexes. ClinSci 2002;102: 639-44.

2. The gonads: Development and function of reproductive system. In: BarrettKE, Barman SM,Boitano S, Brooks HL. editors. Ganong's Review of Medical Physiology. 23 ed:McGraw Hill; 2012; 391-428.

3. Farage MA, Neill S, MacLean AB. Physiological changes associated with the menstrual cycle: A review. Obstet GynecolSurv. 2009;64(1):58-72.

4. Barth C, Villringer A, Sacher J. Sex hormones affect neurotransmitters and shape the adult female brain during hormonal transition periods. Front Neurosci. 2015;9:37.

5. Teichner WH. The recent studies on the simple reaction time. Psychol Bull 1954; 51: 128-49.

6. Kopell BS, Lunde DT, Clayton RB, Moos RH. Variations in some measures of arousal during the menstrual cycle. J NervMent Dis. 1969;148(2):180-87.

7. Smith MJ, Keel JC, Greenberg BD, Adams LF, Schmidt PJ, Rubinow DA, et al. Menstrual cycle effects on cortical excitability. Neurology. 1999;53(9):206972.

8. Baker MA, Weiler EM. Sex of listener and hormonal correlates of auditory thresholds. Br J Audiol. 1977;11(3):65-68.

9. Nene AS, Pazare PA. A study of auditory reaction time in different phases of the normal menstrual cycle. Indian J Physiol Pharmacol. 2010;54(4):386-90.

10. Das S, Gandhi A, Mondal S. Effect of premenstrual stress onaudiovisual reaction time and audiogram. Indian J Physiol Pharmacol. 1997;41(1):67-70.

11. Bhatia S, Tandon OP, Sharma KN. Brainstem auditory andvisual evoked potentials in menstrual cycle. Ann Natl Acad Med Sci. 1991;2:177-82.

12. Bruce J, Russell GF. Premenstrual tension. A study of weight changes and balances of water, sodium, and postassium. Lancet. 1962;2(7250):267-71.

13. Afshan A, Bahulekar A, Swati IA. The influence of different phases of normal menstrual cycle on simple visual reaction time. Int J Bioassays. 2013;2(4):716-18.

14. Yadav A, Tandon OP, Vaney N. Long latency auditory evoked responses in ovulatory and anovulatory menstrual cycle. Indian $\mathrm{J}$ Physiol Pharmacol. 2003;47(2):179-84. 